t! b de marzo, se mervient hachen

do una laparotomía mediana supraumbilical. Los tejidos están acartonados, duros e-invadidos por colecciones purulentas múltiples hasta el tejido celular subcutáneo. Se aspira esta supuración y por un ojal se extrae la sonda. Como es imposible reconstruir planos, se sutura en uno solo peritoneo y aponeurosis, previa colo cación de sulfa local. En el post-ope ratorio se dan antibióticos. La tempe. ratura oscila entre 39 y 37 grados y el 19 sale por completa curación.
Hematíes, 4.670.000.

Leucocitos, 12.400 .

Hemoglobina, $80 \%$.

Valor globular, 0,8 .

Neutrófilos, 76.

Eosinófilos, 3.

Linfocitos, 19.

Formas de paso, 2.

Sedimentación:

A la $1 / 2$ hora, $56 \mathrm{~mm}$.

A la hora, $110 \mathrm{~mm}$.

Glucemia, $0,89 \%$, y

N. P. N., 27 mg. $\%$.

\title{
Conducta obstétrica en las mujeres previamente operadas de cesárea
}

\section{Por el doctor MARIO SANZ ARAOS}

\section{CAPITULO 1}

\section{Conildercueiones generales sobre el parto, en pacientes previamente ope- radas de cesćrea.}

Existe aún una verdadera contro versia en el campo obstétrico en ro lación con la conducta que debe se guirse ante un embarazo a término. en una paciente previamente operada de cesárea, ya que mientras unos sostienen que una operación cesárea no es indicación de una nueva ope ración, otros siguen el conocido atorismo que dice: "una vez una cesárea, siempre una cesárea".

En verdad, la realidad de este alo rismo, podía ser absolutamente cierta cuando se practicaba la cesárea cor- poral sistemáticamenie, sobre todo. teniendo en cuenta el dato que daba Holland, quien decía que el $20 \%$ de las rupturas uterinas eran debidas a - la cescárea corporal.

Pero la técnica moderna de la operación cesárea, es decir, la cesárea segmentaria transperitoneal, ha ablerto nuevos horizontes para el futuro de la mujer con cesárea previa.

El mismo Holland asegura que el $4 \%$ de las rupturas uterinas del emba. razo se deben a la cesárea comporai sin embargo, desde que se viene practicando la cesćrea segmentoria trans. peritoneal, no se ha presentado aun en nuestro medio, un solo caso comprobado de ruptura espontánea del útero, durante el embarazo. Es más, se puede asegurar, que las rupturas 


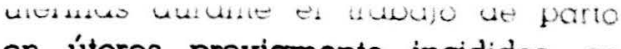
en úteros previamente incididos, se producen únicamente por abandono del trabajo, cuando no por seguir una conducta obstétrica en total désacuer. do con los principios básicos de la obstetricia.

Una de las precauciones que debe tener el obstetra que atiende el tra bajo de parto de una mujer con cesá rea previai, es el indagar - y conocer la historia clínica de la paciente, es. pecialmente los datos concernientes al embararo y al trabajo de parto en que tue intervenida, así como las complicaciones que hubieran podido sur. ofr durante el acto operatorio mismo.

Asi, pues, se debe averiguar por la edad del embarazo, el número de horas de trabajo (si lo hubo) que prece dieron a la operación cesárea, la indicación de ésta y la técnica que se siguió en su ejecución, el peso del ni. ño. la forma en que evolucionaron el post-operatorio y el puerperio, etc.

También es muy interesante aber a entes de la opercación hubo partos a término, y ástós fueron espontá. neos o no, sl después de la operación ha habido partos a término y el modo de terminación de éstos.

Si analizamos detenidamente los puntos anteriormente expuestos hallaremos claramente la necesidad de co nocerlos. El conocimiento del número de partos anteriores a la operación cescrea y su modo de ierminación es de auma importancla, ye que no to puede considerar on las mismas condiciones a una secundigestante con cesdrea previa, que a una multiges. tante con una sola cesárea en su his torial obstétrico, puesto que éstá úttl. met por sus antecedentes tiene mayo. les propaninadades de terminar nor malmente el futuro trabajo de parto. con embarazo a término, y mayores to davía si la operación cesărea le fue practicada on el primer embarazo.

De la micma manera, el número de cesáreas ejecutadas en una paciente. hace disminuír las probabilidades de un parto por via baia en un embara $z o$ a termino.

Esto es tan claro, que podríamos aventurar un principio obstétrico: las posibilidades de un parto espontáneo a tórmino en una paciente previamen. te operada de sesćrea, cumenta en razón directa con el número de partos por vía baja y en razón inversa con el número ae cesćreas.

La veracidad de este "principio" se ve claramente confirmado en la prác tica y en el estudio que haremos posteriormente de algunos casos en el curso de este tema.

Siempre us interescante conocer el número de horas de trabajo que pro cedieron a la opercición cosárea, espe dalmente a constdera el ceiso do una secundigestante, pues si en sus antecedentes ligura que la operación se practicó sin prueba de trabajo o apenas inielándose éste, en realidad es una primigestante desde el punto de vista de las modificaciones que sutre el cuello.

De 21 secundigestomies con cesćrea previa, que terminaton ou eogundo embaraso par vía baia tis inerua operadar aln trabajo provionial acto quirirgitco $Y$ en segundo embaraws tu. vieron una duración promedla da 19 horas y cuatro minutos, durante ol trabajo do parto. En camblo, le resto, o sea i5, que habian tenido trabajo pre vio a la operación ceadma, ca ol ce. 
gundo embarazo tuvieron una duración promedia de 13 hora's y dos minutos en el trabajo de parto

Conocer la indicación de la operación cesárea anterior, es de capital importancia para deducir la conducta a seguir en un trabajo de parto ul. terior con embarazo a término. Es na tural que no se tendrá la menor duda al respecto de la terminación de un trabajo en una paciente operada an. ies por estrechez cubsoluta, así como se deben guardar grandes esperanzas en la terminación espontánea y natu. ral de un trabajo de parto en una paciente operada anteriormente por una complicación del embarazo mismo, o del parto, como una toxicosis o una procidencia del cordón

Pero tal vez, la mayor preocupación del obstetra es conocer el estado de la cicatriz uterina, su resistencia ante la distensión que le impondrán la contracción y retracción uterinas durante el período de dilatación y especial. mente durante el de expulsión. Des. graciadamente no se han encontrado los medios precisos de información so. bre este estado, y sólo llegamos a sospechar la calidad de la cicatriz uterina por los antecedentes del puer. perio y por un examen que, por más cuidadoso que sea, sólo nos da ideas muy vagas de su consistencia.

Es natural que en el sitio de la cicatriz uterina la resistencia sea menor, puesto que un tejido flbroso poco extensible ha reemplazado las tibras elásticas del segmento. S1 el post-operatorio tue tebril, o hubo supuración de la clcatriz uterina podemos tener la seguridad de que ontre el tejido fibroso de reparación se ha interpues. to telido confuntivo y que por tanto la cicatriz de la herida uterina no nos dará garantía suficiente $a$ un trabajo ligeramente prolongado o a una prueba de trabajo. Para algunos autores cmericanos, el solo antecedente febril del post-operatorio es indicación de una nueva cesárea.

Por otra parte la implantación de la placenta en el sitio de la cicatriz viene a disminuir la consistencia de ésta, y entonces se tiene uná causa coadyuvante de la ruptura-uterina. Algunos tocólogos ante esta eventua lidad proponen que, sistemáticamen. te, en toda paciente con antecedente de cesárea y con un embărazo a término, debe tratar de visualizarse la placenta por medio de los Rayos X.

Pero en general, la cicatrización de la herida uterina se hace en forma tan perfecta, que al Instituto de Protección Materno-Infantil se han presentado pacientes con muchas horas de trabajo y con una integridad uterina per. fecta, como el caso insólito, aunque veríaico, de la historia número 1.281 . Servicio del Protesor Molica, de 1951. que demuestra que la paciente suje to de esa historia estuvo 53 horas en trabajo de parto, el cual terminó por medio de una aplicación de fórceps por trabajo prolongado. Notable tam bién es el caso de la historia número 2.208 del Servicio del Profesor Acosta (1950), que con antecedente de cesá res por estrechez, tuvo un trabajo de 33 horas 50 minutos y terminó en una aplicación de fórceps con feto muerto. Esto, pór no citar sino dos casos que nos demuestren claramente la calidad de la cicatriz que se forma, des. pués de una operación cesćrec.

Es necescrio tener en cuenta que al trabajo de parto de una pacienle 
con cesárea previa, no se realiza ge neralmente en una forma normal, qui- zás porque la cicatriz uterina viene a ser una espina irritativa que trastorna la dinámica 0 una barrera quie al tras. tornar la arquitectura uterince, sirve de obstáculo para la repartición simétri. ca del estímulo contráctil. Asi, de 132 pacientes que hicieron su trabajo de parto en el Institulo de Protección Malerno-Infantil, con antecedente de cesárea previa, un $67 \%$ hizo un trabajo irregular y sólo un $33 \%$ un trabajo satisfactorio:

Es necesario también examinar detenidamente a la paciente, ya que sólo el examen puede permitir cono cer la conductc obstétrica indicada. su trabaio de parto, puesto que muchas veces la edad del embarazo no permite otra conducta que la expectativa. Una de las pacientes que ingresó al Instituto con antecedente de dos cesćreas por estrechez relativa de la pel. vis, tuvo un parto espontámeo, cunque con teto premeturo, pero vivo.

Por regla general, el examen gene. ral obstétrico no varia en ningún punto del que se le debe hacer a toda mujer embarazada: y en trabajo de parto.

Fara mayor claridad de las conductas obstétricas y de sus resultados, he mos dividido el trabajo que presentamos, según las distintas conductas obstétricas, en relación con el antece dente y la incicación de la cosárea anterior.

Así, pues, estudiaremos los casos de parto espontáneo, aplicación de fórceps, la extracción podálica, la versión interna y la extracción podálica, y fincimente los casos en que se htzo una nueva cescrea $Y$ sus indicaclones.
CAPITULO II

Tarminacibn del parto on las paciontes provilamonte oparadas de ceiderea.

\section{I - PARTO ESPONTANEO}

La base fundamental para lograr éxito en la: atención de un parto en una paciente con cesárea previa, se deriva única y exclusivamente de dos factores, a cual más importantès: un examen cuidadoso y una vigilancia constante.

Por medio del excmen, podremos anatizar, juzgar y deducir las posibilidades de un parto por vía baja o la necesidad de intervenir directamente para salvar la vida del feto y no pocas veces la de la madre.

$\mathrm{Si}$ en condiciones normcies el tra. bajo de parto de cualquier paciente, debe ser rigurosamente controlado, so pená de tener tracasos rotundos, con mucha mayor razón debe vigilarse el trabajo del parto de una paciente que ha sido anteriormente operada de cesćrea. Podemos sostener, sin lugar a equivocarnos, que la peor contingen. cla que le puede suceder a un obstetra que se encuentre alendiendo un parto en una paciente operada de cesárea, cual es la de la ruptura uterina, se debo únicamente a descuido, cuando no, como dijimos anteriormente, a una condmete fuera de lóolca.

Poro no colamente el trabajo de parto debe ser culdadosamente controlado por el pellgro de contratiem. pos descagradables, sino también para dirigirlo, ya que en muchas ocasio. nes, en esta clase de pacientes, el tra. bajo ce denarrolla on une forma tan trancamente frovilar, que at no or 
llevado pronto a la normalidad, puede dar origen a conductas lesivas para el feto o la madre o para ambos, cuando no viene a pagar el feto con su vida un descuido del obstetra.

Por otra parte, durante la vigilan. cia del trabajo de parto, podemos en mendar errores que se hubiesen podi: do cometer durante el excmen inicial. El parto espontáneo, en una paciente con el antecedente de una cesárea nrevia, es posible.

De las 132 pacientes operadas an. tes de cesárea, que ingrescron al servicio en los años de 1950 y 1951, tuvierón parto espontáneo 51 de ellas, lo que reducido a datos estadísticos. da una incidencia del $38.62 \%$.

El mayor número de partos espon táneos correspondió a aquellas pa. cientes que habian sido operadas por la indicación de desproporción cefalopélvica, annque hay que tener en cuentc que este grupo es el de mayor proporción. De 64 pacientes, 16 tuvieron un parto espontóneo, 13 con feto a término, y tres prematuros, con una mortalidad fetal de uno, por prematurez.

De las 15 pacientes que ingresaron con antecedente de cesárea por es. trechez pélvica, tres tuvieron un parto espontáneo, una con feto prematuro y dos con embarazo a término; po ro con feto muerto y macerado, 10 que explica porque i cabaiga. miento óseo de los huesos de la cabeza fetal hizo que los diámetros de esta fueran proporcionales a los de la pelvis materna.

Podemos asegurar que no existiendo la causa que originó la cesdrea anterior, y no hablendo otra indica. ción para una nueva ceedroes trata pierde su vigencia en un nuevo embarazo.

Así, las pacientes que habian sido operadas de cesárea por toxicosts del embarazo, cinco entre ocho, tuvieron un parto espontáneo, cuatro con em barazo a término $y$ una con un feto prematuro, todas con feto vivo y $\sin$ complicaciones durante el puerperio.

De slele pacientes que habian sido operadas por anomalías en la presen. tación fetal, tres tuvieron en nuevo - mbarazo, con parto espontaneo, on las cuales dos con toto a termino $y$ una con un embarazo gemelar, las cuales salieron del servicto con sus niños vivos y en buenas condiciones.

Con antecedente de cesćrea por sufrimiento fetal y falta de encajamiento, acudieron al Instituto de Prolección Materno-Intontil cuatro pacientes, todas con embarazo a término y entre éstas, dos tuvieron un parto espontá. neo con un trabajo regular y con re. sultados satisfactorios, pues los niños no presentaron ni morbilidad y menos mortalidad ninguna $y$ on las que el trabajo de parto se verlficó con una duración promedia de 12 horas; de las otras dos pacientes, una terminó su parto con fórceps y la otra con ce. sárea.

Pero el grupo que más llustración nos da sobre las grandes probabillida. des que tienen actualmente las operadas de cesćrea de no ser Intervent. dos otra vez, es el de aquellas pacion. tes que en el curso de su historiálabs. tétrica sufrieron una operación cesdrea por inserctón baja de la placenta. Once pacientes Ingresaron con exte antecedente y las 11 tuvieron un par. to por via baja, nueve do ellas espon. t́́necumente y dos por medio de apllcoctón de tórceps. 
En estas nueve pacientes que parieron espontáneamente, ocho habían llegado a la terminación de su embarazo y sólo una-tenía un feto prematuro.

El trabajo tue de una duración pio media de 21 horas con 13 minutos, 10 cual quiere decir, que si- hubo pacien. tes que hicieron un trabajo de parto en 12 horas, también nos enseña que las hubo con duración de más de 30 horas, trabajo exagerado para una paciente en condiciones normaies $y$ por lanto, sumamente peligroso en una paciente con un antecedente de cesćrea.

Pero por otra parte, eso también nos puede dar idea de la calidad de cicatriz que se forma en algunos úte ros, con garantía para varias horas de trabajo.

Entre estos fetos hubo una mortalidad de uno por anoxemia, ocasiona: da por una procidencia del cordón, y una morbilidad fetal también-de una unidad, por sutrimiento fotál, ocasio. nado por trabajo prölongado.

Investigando los antecedentes de las 132 pacientes, en 15 de ellas no se logró dilucidar con claridad la indicación por la cual sufrieron la opera ción cesćrea, pero de śstas, 10 fue ron consideradas en capacidad de ter. minar su parto espontáneamente, $y$ así fue: todos con teto vivo, arrojando un promedio de 11,20 horers de trabajo: nueve fetos a término y uno prematuro. La mortalidad y la morbilidad en este grupo tue de cero (0).

Entre las 51 pacientes que terminaron su trabajo de parto espontáneamente. una tue inducida. Aunque no es sino un caso nos demuestra que se puedo Inducir el trabajo de parto en una mu- jer con cesárea prević, extremando el control hasta la exageración durante todo el período del parto. Esta inducció tue ejecutada en una de las dos pacientes que ingresaron con antecedente de cesárea por éritroblastosis fetal, y que además sutría de una dia. betes. por lo que el producto de su concepción pesó 5.120 gramos, a pe. sar de no contar el embarazo sino ocho meses. Con tratamiento adecua. do, exsanguino-transfusión, vitamina $K$, etc., el niño salió en buenas condi. ciones del Hospital.

El promedio general, en horas de trabajo, de las pacientes que terminaron su trabajo de parto espontáneamente tue de 16 horas 20 minutos, pero el $46 \%$ de los trabajos de parto fueron irregulares, dando ocasión a que el médico intervinierc directamente para modificar el trabajo por me dio de sedantes. Esta cífra es en realidad bastante alta, y como la unica diferencia entre estas pacientes y las que no han sido operadas es la cicatriz uterina, tenemos que concluír 16 . gicamente que-ésta sirve de punto irritativo que viene a desordenar la regutaridad de las contraccioners uterinas.

Entre estas pacientes, se presentaIon dos casos de morbilidad fetal, con signos inequívocos de sutrimiento to tal, por trabajo prolongado y una por eritroblastegis.

Hubo cuatro casos de mortallidad fetat, uno nacido muerto por cmoxemia durante el parto, debido a procldencia del cordón, otro, muerto recién nacido por prematurez y dos nacidos muertos y macerados, uno de los cuas. les por anemia de la madre y palu. dismo. 
los resultados de los partos espontáneos, en pacientes con cesárea previa, tenemos:

\begin{tabular}{|c|c|}
\hline spontáneos $\operatorname{con}^{-} \operatorname{ce}$ & \\
\hline previa & $38.62 \%$ \\
\hline Con embarazo a érmino & $82.35 \%$ \\
\hline Con feto prematuro ... . . & $17.84 \%$ \\
\hline Promedio en horas de trabajo & $16.20 \mathrm{ttrs}$ \\
\hline Trabajo regular en .. & $54.00 \%$ \\
\hline Trabajo irregular en & $46.00 \%$ \\
\hline Morbilidad letal . . & $5.76 \%$ \\
\hline Mortinatalidad ........ & $5.88 \%$ \\
\hline Mortalidad Neo-natal $\ldots$.. & $1.96 \%$ \\
\hline
\end{tabular}

\section{II - FORCEPS}

Los rotundos tracasos derivados del abuso del fórceps y de su áplicación indiscriminada, han hecho que en la obstetricia moderna se fijen categóricamente sus indicaciones y se mantengan vigentes las condiciones materno-fetales quie lo hacen-benéfico para la feliz culminación de un em. barazo Olviderilas sería correr el tre mendo riesgo no sóto para lo madre $y$ el têto aistadamente, sino en con. iunto, además de que una errónea intervención pone en la picota del desprestigio a quien por carecer de un buen criterio obstétrico se lanza a aplicar un fórceps sin ánimo sereno, y $\sin$ severo conocimiento de la técnica.

Indicaciones y condiciones para el empleo de tórceps son de todos los abstetras conocidas y no tendría ob: jeto enumerarlas en este trabajo.

Nos detendremos sí, a estudiar muy someramente el punto que puede interesar a este trabajo, esto en, el capítulo del tórceps llamado indicación profilácetiea. ncista ayer no mas, la indicacion profiláctica del fórceps se consideraba única y exclusivamente por el lado materno; hoy, algunas escuelas sostienen que debe contemplarse tam. bién el aspecto fetal pensando en el posible menor traumatismo que sufri. ría un prematuro extraido con fórceps. Nosotros consideramos que el uso profiláctico del fórceps debe re servarse para evitar complicaciones que pueden ser de orden local o ge. neral.

Las indicaciones de orden local plantean al obstetra, especialmente on las estrecheces límites y en las resis. tencias anormales del piso pélvico. Pero debemos estudiar una contingenlia no analizada aún en detalle; y es el empleo profiláctico del fórceps en la mujer previamente operada de cesárea.

Anteriormente decíamos que el mayor peligro que puede atrontar el obstetra, es la ruptura de la cicatriz seg. mentaria en el trabajo del parto, pelioro-que adquiere proporciones inmensas durante el período de expulsión. en comparación con las que pudieron existir durante el periodo de dilación.

Durante el periodo de expulsión, el peligro de una ruptura puede evitarse con la aplicación del tórceps, tórceps profiláctico, puesto que no va a servir de trortamiento sino preventivo. En suma, sería un fórceps profiláctico de desprendimiento por alleraciba lo cal de orden materno.

Sin considerai como indicáción po réntoria de aplicactón de fórceps, el antecédente de cesćrea, tenemos que admitir que cualquiera otra indicactón cobra mayor valor por el misno ante oedente. 
Sobre los 132 casos que estamos estudiando, se hizo aplicación de fór* ceps en 22, a sea en el $16.66 \%$, cifra relativamente baja, si tenemos en cuenta el número de partos por las vias naturales.

Sólo en seis casos se hizo aplica ción de forceps por el antecedente de la paciente, to que puede explicar en parte, el número de tórceps aplicados por otras indicaciones. El trabaio pro. longado es una de ellas, porque en estas pacientes el trabajo es generalmente irregular y así no sólo se alarga, sino que por consiguiente, origina el sufrimiento fetal, otro motivo de coplicación de tórceps.

Entre los 22 fórceps, tres de ellos fueron aplicados por sobre el estrecho superior y aunque los niños salieron en relativas buenas condiciones del servicio, uno de ellos sufrio una hemorragia meníngea y otro neumopatía por sufrimiento fetal. De las tres aplicaciones de forceps clto, dos se ejecutaron bajo la indicación de sufri. miento fetal. Las tres pacientes habian sido operadas antes por despro. orción cetalo-pélvica.

Lejos estamos en este trabajo, de -omar armas en pro de la aplicación de fórceps alto, o ir en contra de ellat ya oque no es materia de él; nos limitamos solamente a anotar sus conse. cuencias, lo mismo que de cualquier conducta obstétrica; por otra parte, ya está demostrada la gran incidencia de hemorragias meningeas on los tetos extraídos con tórceps alto y los pell. gros de ruptura uterina, máxime existiendo una cicatriz de cesćrea.

Se encuentra on las pacientes ope. radas bajo la indicación de despropor. ción celalo-pélvica, la mayor inciden. cia de aplicación de tórceps, ya que de 64 pacientes que ingresaron con este antecedente, 12 tuvieron un parto por vía baja, pero con aplicación de fórceps, lo que da un porcentaje dal $18.75 \%$, de los cuales uno correspon. dió a un prematuro $y$ el resto a fetos a término, tres por trabajo prolongado. dos por sufrimiento fetal, dos por in. dicación profiláctica y uno por sufrimiento fetal, precedido de distocia de! cuello. La aplicación de fórceps altos correspondió también -a este grupo.

El promedio total en horas de trabajo en este grupo de pacientes es bastante más alto que las que tuvieron su parto espontáneamente, con el mismo antecedente de cesárea por desproporción cefalopélvica. La morbilidad estuvo limitada a los dos fetos que fueron extrcídos con aplicación de fórceps alto. Hubo una muerte del re clén nacido por hemorragia meníngea.

El promedio de duración del trabajo fue de 26 horas 53 minutos.

Un detalle interesante es el de que en ninguno de estos casos se presentara un solo trabajo- regular, contribu yerido en esta forma al trabajo prolon gado y al sufrimiento fetal.

El resultado de las aplicaciones de fórceps a las pacientes que ingresaron con antecedentes de cesárec por estrechez pélvica no pudo ser más desastroso. Hubo dos aplicaciones de fórceps: una, en un feio prematuro que después acusó morbilidad por sufrimiento fetal, y otra, en un felo a tér: mino, el cual murió durante la aplicación del aparato obstétrico.

El promedio en horas de trabajo. ontre estas dos pacientes, fue de 35 horcs 17 minutos, promedio que aclara ampliamente el accidente. En deti. 
to le punio usanamado que intento remediar una aplicación de fórceps.

Desgraciadamente no todas las pacientes que ingresan al Instituto de Protección Materno-Infantil, obran prudentemeñte internándose con la iniciación de los dolores, sino que muchas acuden a solicitar servicios en condiciones bastante precarias, desde el punto de vista tanto materno como fo tal. lo que explica los altos índices de morbilidad.

Se hizo aplicación de fórceps en una de las pacientes que ingrescton con antecédente de cesárea por sufrimiento fetal $y$ falta de encaiámiento. El puerperio en la madre fue fébril. No es el caso de discutir en este tra. bajo el criterio que se tuvo para elo gir una conducta en pácientes con tail antecedente.

Del grupo de pacientes que ingresa. ron con el antecedente de cesárea por toxicosis gravidica, se hizo aplicación de fórceps en un solo caso por el antecedente de cesárea, con-resultados satisfactorios.

En dos pacientes que llegaron con trabajo de parto y con antecedente de cesárea por presentación distócica irreversible, se hizo aplicación de lórceps, una de ellas por falta de rotación $y$ demora en el descenso de la presentación, y-la otra por el antecedente de cesćrea. El promedio en horas de trabajo en estas pacientes tue de 12 ho ras 15 minutos y los resultados am pliamente satisfactorios.

Con un promedio un poco alto en horas de trabajo, se hizo aplicalión de-lórceps en dos pacientes que tonian un antecedente de cesárea por inserción baia de la placenta, siendo ceps el sufrimiento fetal.

El promedio en horas de fue 25.57 horas, siendo éste t en ambos casos, presentánde. caso de morbilidad en uno de to ños, por sufrimiento fetal.

Con la aplicación de lórceps a grupo de las pacientes que tnot. ron con antecedente de cesárea inserción baja de la placenta, wo pletó el total en partos por vie puesto que 11 pacientes que 1 . ron al Instituto con este ante. nueve tuvieron un parto eapest. las otras dos to tuvieron con e. ción de fórceps.

En las 15 pacientes en quibi se pudo averiguar la indicación cesárea, se hizo aplicación de thi. a dos; a una, por trabajo prolon: y a la otra por sufrimiento fetal, 2 mero promedio de horas es el me. de todos, siendo de 35 horas. minutos y se presentó morbllick uno de los dos fetos, por sufintat fetal y traumatismo obstétrico.

Salta a la vista el hecho do morbilidad fetal tue muy alta, to nós hace pensar qué existió en resolver la conductci obita presunción relativamente cferta, considerán los índices tan altos horas de trabajo.

Sintetizando en un cuadro los datos relativos a la aplios. írcepe en pucientes con bectir via, tenemos:

Partos con aplicación de fór.

Partos a término: 20 . Partos promaturos: 2 . Fórepos en la excavaciónt 19 
$10.00 \%$

Fu sutimento fetal: 10 . . $44.99 \%$

Put cescrea previa: $6 \ldots \ldots 27.27 \%$

- Par trabajo prolongado: 5 . $22.70 \%$

Por talta de rotación y des.

cense de la present. . .. $4.09 \%$

Motbllidad fotal ... ... . . . $18.18 \%$

Matnlided tetal ..... . . . $9.09 \%$

bit tramalismo obstetrico: 2

extas:

Aublidat materna: I caso:

puotpertio subtebril ...... $4.09 \%$

Heras de trabajo promedio $25.32 \mathrm{H}$. Trabalo trregular en el .... 84.62\%

\section{III - CESAREA}

En páginas anteriores decíamos que el número de cesáreas hacía dis. stinuit las posibilidades de un parto fort via baja, lo cucil es evidente, por. que a mayor número de cesáreas, ma. yat numero de cicatrices y por tanto. mayor número de puntos débiles utemosi

Sin umbargo, en el Insttuto de Pro. - lecetón Materno-Intantil, de las 56 pa. ctentos que fueron nuevamente opera. des de cesárea sólo en una paciente In indicación de la nueva cesárea tue - intecodente de cesáreas anteriores. que habian sido tres, dándose el aso en cambio, de una paciente que Eenfa ol antecedente de cuatro cesdzecs tuvo un parto por vía vaginal y nof modio de aplicación de fóncops. Is tudictón de las cesáneas on el tento de las paciontes tuvo otra indi. sactón.

De las pactentes con antecedentes de cusárea por desproporción cetalo. pelviea. hueron nuevamente operadas 34. 29 de ollas fueron nuevamente in. wrventader por la untama indicación anterion: por sutrmento felal y lalta de encajamiento: tres: por procidencia del cordón, una, y por antecedente de tres cestreas, una.

El promedio en horas de trabajo en este grupo de pacientes, tue de 25.20 horas aproximadamente $y$ sólo un $15 \%$ hizo un trabajo regular.

Se presentó morbilidad fetal en dos casos, ambos con signos de sufrimien. to felal.

De 18 pacientes que ingresaron con el antecedente de operación cescrea. por estrechez pélvica, 12 terminarónsu trabajo de parto pos medio de opo ración cesćreci por la misma indicación y después de un total de 28 horas 25 minutos de trabajo.

Ninguno de los letos presentó morbilidad y tampoco se presentó ningún caso de mortalidad.

A una de las cuatro pacientes que ingresaron con antecedente de cesá. rea por sufrimiento fetal se le practi. có nuevemente operación, por dieto sia dindrmica-corporocervieal y falta de encajamiento, previo intento de tratamiento médico con sedanies y an. hespasmódicos. El niño nació vivo $y$ no presentó morbilidad.

Ocho paclentes se presentaron con antecedente de cesárea por toxicosis cravidica y a una se le practicó nue va intervención por sufrimiento fetal y falta de encatamiento, después de 13 horas de trabajo. La causa dal intirimiento wan dos circuiares del oor. dón. No hubo motbilidad ni morbll: dad total.

A dos pactentes aue tenian anteco. dente-de cesdrea, por presentación distócica irneveraible, se les hizo nue va operactón quirúrgica; a una por

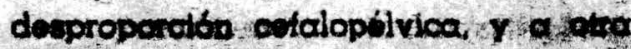


por presentación de hombro, previo intento de versión externa e interna en la segunda. Posteriormente comentaremos las consecuencias de esta conducta.

El promedio en horás de trabajo en estas dos pacientes fue de 20 horas 15 minutos, y no se presentó ningún accidente materno ni fetal.

Con antecedente de cesárea por pri. migravidez en crñosa, se encuentran en las historias revisadas, dos casos, los cuales en un segundo embarazo hubieron de ser nuevamente interve. nidos, por presentación de pelvis incompleta uno, y el otro por distocia cervical.

En estos dos casos, no se presentó morbilidad nii mortalidad materno-fetal y el cómputo en horas de trabajo fue de 26.

La eritroblastosis feta'l es indicación de operación cesárea siempre que fracasen los intentos de inducción del trabajo de parto, cuando esto ha sido indicado por la evolución del proceso de isoinmunización. Vimos anteriormente que se habian presentado dos casos de pcicientes con antecedente de cesárea por eritroblastosis tetal; vimos también cómo una de esas pacientes hizo un parto espontáneo des pués dé una inducción, de cómo ese niño, fruto de una diabética y que pe. só al nacer 5.120 gramos, pudo-ser salvado por medie del tratamiento mé dico. pues bien, en la otra paciente, hubo necesidad de recurrir nuevamente a la operación cesárea, antes de la iniciación del trabaio de parto, de acuerdo con las indicaciones usadas én estos carsos, y a pesar do los estuerzos por salvarlo, el niño talleció poco después de su nacimiento.
Los resultados disimiles de la interrupción del embarazo nos hacen preguntar: ¿estará plenamente justificada la intervención por medio de cesárea? En todo caso, cualquiera que sea la respuesta a este interrogante, la in. terrupción del embarazo por medios quirúrgicos sólo debe ejecutarse cuan. do haya fracasado toda tentativa de inducción del trabaio de parto.

A mi modo de ver, más justificado se encuentra la intervención en-los casos de muerte habitual del feto, -siempren que fracasen los sistemas inductivos del parto.

Al Instituto sólo llegó una paciente coñ este antecedente, la cual tuvo que ser nuevamente intervenida, después de varios intentos de interrupción médica del embarazo. La operación se ejecutó sin accidentes materno-fetales.

Se hizo también cesárea iterativa en una paciente que ingresó con el antecedente de fistula vesico-vaginal operada, que había sido intervenida en el embarazo anterior por la misma - razón. Las plastias vesico-vagináles son indicación de cesárea, ya que es mucho más difíchl obtener éxito en una intervención de esa naturaleza, que lograr el pleno restablecimiento de una paciente con operación cesárea, y muy probablemente el permitir un pcrto por via baja, generalmente implica el restablecimiento de la fístula. Lq paciente correspondiente a este caso, no tuvo sino muy poco trabaio de parto-y los resultados de la operación fueron satisfactorios.

De las 15 pacientes en las cuales no se pudo averiguar la causa de la ce. sćrea previa, se practicó nueva cesá. rea en una de ellas, por presentación de hombro con ruptura prematura de 
is membranas. Se presentó al Institulo con trabajo de parto, después de nueve horas de iniciado y con contrac. ciones intensars. Inmediatamente se procedió a ejecutar la operación quirúrgica, la cual fue hecha sin compli. caciones.

No se presentó morbilidad tetal, y la madre salió en buenas condiciones del servicio.

Entre este grupo de pacientes que fueron nuevamente intervenidas - por cesárea, la indicación de la operación - fue la misma de la canterior en 45 de ellas.

Reduciendo a datos estadísticos, 10 que hemos visto en las operadas de cesárea, tenemos:

Partos intervenidos por cesá-

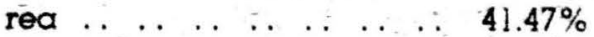

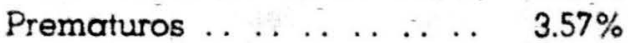

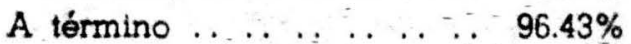

Por desproporción cefalapél.

vica . . . . . . . . . $53.57 \%$

- Eatrechez pálvica . . . . 21.41\%

Sufrimiento fetal y falta de

encajamiento. . . . ...... . $7.14 \%$

Presentación de pelvis incom.

pletc en secundigestante

cñosa .. . . . . . . . . . . $\quad 1.78 \%$

Por presentación de hombro

Procidencia del cordón .. . .

Distocia dinámica corporocer-

vical . . . . . . . . . . $1.78 \%$

Distocia cervical ... . . . . $\quad 1.78 \%$

Entroblastosis fetal ...... $1.78 \%$

Muerte habitual del feto ... $178 \%$

Plastia vesico-vaginal $\ldots \ldots \quad 1.78 \%$

Gescrea previa ........ . $1.78 \%$

Horas de trabajo: $18 \mathrm{H} .10 \mathrm{M}$.

Trabajo regular en .. .... $18.18 \%$

Trabajo Irregular en ... . . . $81.82 \%$

Morbllidad fetel ....... $3.57 \%$
Por sufrimiento felal: 2 casos

Mortalidad fetal . . $\quad \ldots \quad \ldots \quad \ldots \quad \ldots \quad 1.78 \%$

Por eritroblastosis tetal: I caso

Com̄o vemos por et cuadro, una sola de las pacientes tue somefida a una sola intervención por el solo he. cho de tenet un cnteredente de tres cesáreas.

El antecedente de cesárea por sí, muy pocas veces puede ser indicación de una nueva cesárea.

Entre las pacientes que estudiamos, se encuentran 10, que, con el antecedente de tres cesáreas por 10 menos. fueron sometidas a un trabajo de parto. De ellas, cinco tuvieron su parto por vía baja y una con el antecedente de cuatro cesáreas, terminó su nuevo embarazo por una aplicación de fórceps con feto a término y vivo.

Personalmente opinamos que ta indicáción de cesárea basada solamente en el antecedente de una previta. mente ejecutada, sólo la puede dar un examen cuidadoso de le historia clínica y de las condiciones de la pa. ciente.

Algunos autores aconsejon la historectomía o ligadura de trompas, des pués de la cuarta cesárea, con el fin de suprimir la actividad generatriz de la paciente y en esta forma evitarle futuras complicaciones.

En el Instituto de Protección Materno-Intantil no so le hizo esterilización a ninguna paciente; por este motivo, y adelantando conceptos, podemos aso. gurar que el mayor o menor númeto de cescureas no justifica por sí sólo una determinactón de tal calidad.

Los casos de ruptura uterina que se verificaron en el orupo de pacientes que hemos tomado para este estudio. 
sólo se sucedieron en pacientes que no habian sufrido sino una sola operación-cesárea, dándose en cambio. el caso de que a una de las 135 pacientes se-le-practicó la sexta cesárea y se dejó el útero.

Por tanto, si el número de cesáreas es indicación de esterilización, por los casos estudiados tendrímos que ad mitir que ésta debería practicarse con la primera operación.

Rechazamos, pues, catégóricamente. la esterilización que tenga como única indicación el número determinado de operaciones cesáreas.

\section{IV - EXTRACCION PODALICA}

La extracción es una maniobra obs: tétrica destinada a terminar rápidamente un trabajo dé parto con un feto en presentación de pelvis, estando su indicación limitado al sutrimiento fétal o al agotamiento materno.

Casi_podríamos decir que la extracción podálica es a la presentación de pelvis, lo que la ajplicación de fórceps es a la presentación celálica, Hevan. do más riesgoo, claro está, la primera de las conductás siendo la incidencia de hemorragias meníngeas fetales barstante alta, especialmente en los prematuros, así como las fracturas de los huesos del cráneo en la extracción de la cabezá fetả.

Por otrá parte, la extracción podá. lica con el cuello incompletamente dilatado, tíene el riesgo de la retención de la cabeza telal, o por la misma fuerza de tracción, los brazos fetales suelen no sequir el movimiento dal cuerpo fetal quedando retenidos con la cabera última, Cuando sucede esta contingencia no es raro ver fracturas de los miembros fetales al tratar de descenderlos. También puede ocurrir desgarraduras del cuello uterino.

La aplicación de fórceps tiene la ventaja sobre la extracción podálica, que en aquélla hay garantía suficiente sobre la relación pelvitetal, ya que la aplicación de fórceps se debe hacer sobre la cabeza encajada: en cambio. en la extracción podálica la relación de los diámetros de la cabeza letal con los diámetros pélvicos maternos, no se puede predecir.

Es en definitiva una conductc peligrosa y a menos que las circunstancias lo exijan, la evolución de un parto a término y con proporción pelvifetal, siendo la presentación de pelvis, debe dejarse en su curso nattural.

En el número de casos que estamos estudiando, sólo se encuentra un parto con feto de $8 \frac{1}{2}$ meses, obtenido por extracción podálica, en una paciente que fenía antecedente de cesárea por estrechez pélvica.

La conducta adoptada por sufrimiento fetal no surtic los beneficios esperados, pues hubo retención de cabeza última, con la consiguiente muer. te fetcil.

El trabajo de la paciente había sido irregular y el peso del niño fue de 2.420 gramos.

La conducta en sí es discutible, porque además dé los peligros inherentes a la extraccion pociálica, habria que tener en cuenta, no sólo el ante cedente de cesárea, sino el grado de estrechez, lo que hacía presumir una gran dificultad en la extracción del polo cetálico.

Si-es en suma una conducta pellgrosa cuando hay o se presume una adecuada proporción pelvi-fetal con 
mayor razón lo es en casos de estre. chez pélvica aun con feto prematuro y con la presencia de una cicatriz seg. mentaria.

\section{$\checkmark$... VERSION INTERNA $Y$ RUPTURA UTERINA}

En las primeras páginas de este tra bajo asegurábamos que la ruptura uterinci en pacientes con embarazo a término y con antecedente de cesárea somentaria, se sucedía únicamente o por abandono en el trabajo de parto fcausa común de las rupturas uterinas), o por la adopción de un conducta reñida con los principios obstétri. $\cos$.

He querido intitular este capítulo en la forma en que lo hice, no porque con la versión interna se hayan pro ducido todas las rupturas uterinas, en et grupo que estamos estudiando, pero si porque todas las versiones internas Frodujeron rupturas uterinas. IGra cias a Dios, que no fueron sino dosl.

En el curso de este trabajo, hemos venido insistiendo en la relativa resis. tencia del llamado punto débil del útero histerotomizado. Pero no podo mos negar que al quebrarse la arquitectura uterina, mal reparada por una neoformación fibrosa, quedan puntos de menor resistencia.

La cicatriz de la herida semmente. ria, que soporta grandes tuerzas de tracción, como lo hemos visto, es débil para soporkar las fuerzas que la deforman.

Esto por lo menos nos lo da a en. render el hecho de que en las dos oca siones en que se hizo una versión in terna, se produjeran rupturas uterinas.
A riesgo de ser llamados absolutis tas, pretendemos que la versión in. terna en unc paciente con embarazo a término, cualesquiera que sean las circunstancias en que se presente el trabajo de parto, es una conducta de consecuencias tan peligrosas que su uso, debe en lo posible, proscribirse.

Con cuánta mayor razón no recha: zaremos esta conducta en pacientes que han sufrido una intervención de la calidad de una operación cesárea.

Su contraindicación en estos casos. no-puede estar más demostrada, tánto por la teoría como por la práctica.

Las dos versiones se verificaron en dos de aquellas pacientes en quienes no se pudo averiguar la causa de la cesárea, ambas con un solo antece dente de cesárea, la una secundigestante y la otra con el antecedénte de varios partos espontáneos. Las dos versiones lal decir versión en este caso lo hacemos sinónimo de ruptura uterinci), se verificaron sobre feto vivo a lérmino, por presentación de hombro.

La mortalidad estuvo limitada úni. camente a los fetos, ya que por la ra. pidez de la intervención se logró salvar la vida de las madres.

Hemos ancilizado a grandes rasgos, lo que llamamos conducta "ilógica", $y$ hemos visto sus resultados. Analicemos ahora la otra causa común de rupturas uterinas.

Habiamos dicho anteriormente que una de las exigencias en la atención de un trabajo de parto en una paciente con cesárea previa era una vigilan. cia permanente y racional.

Una de las pacientes que llegó con antecedente de cesárea por toxicosis gravidiea, tuvo un abandono en su trabajo de parto de $4 \frac{1 / 2}{2}$ horas, tiempo 
suficiente para que se produjera una ruptura uterina;-solución de continuidad que se verificó por la cicatriz de la cesárea prévia, que prolongándose interesó la arteria uterina derecha.

El trabajo de parto en esta paciente habia sido relativamente corto, . de ocho horas; desgraciadamente la his toria de la paciente por el mismo abandono del trabajo, no menciona lo interisidad de éste.

No hubo mortalidad materna, ya que la paciente fue intervenida a tiempo. El feto era a térmíno y pesaba 3.700 gramos.

Otra paciente-con antecedentes de operáción cesárea por desproporción cefalopélvica, alcanzó a llegar en su trabajo de parto a la dilatación completa, después de una labor de 25 heras, pero la resistencia de la-cicatriz uterina cedió, estableciéndose en esta forma una amputación casi total del cuerpo del útero, siendo necesario completarla quirúrgicamente.

El teto pesaba 3.740 gramos y ha bia llegado vivo al Servicio. La ma dre salió del Instituto por restablecl. miento.

La causa de esta ruptura uterina aunque se verificara por el sitio de la cicatriz fue debida al olyido a que se sometió la enferma.

El último caso de ruptura uterina que se presentó fue el de una multigestante que había ingresado al lnstituto con un aniecedenie de cesáred por desproporción, en su primer embarazo.

La paciente ingresó con un embarazo a término cón feto en presentación de pelvis y ruptura prematura de las membranas; lo que dió origen a un trabajo intenso e frregular. Esta pa clente fue sometida a control directo hasta completar una dilatación de seis centímetros, - y entonces "fue olvidada" por un período de cuatro horas en que se-encontró a la paciente en estado de shock $\mathrm{y}$ con "una versión es pontánea" de la presentación.

A pesar de los intentos por restable cer a la paciente para someterla a in tervención quirúrgica, tallecio dos he ras después.

En resumen tenemos:

Rupturas uterinas, $2 \ldots \ldots \ldots, \quad 3.70$

Por versión interna $\ldots \ldots \ldots \ldots 40.00$

Por abandono del trabajo de

parto ... ............

60.00

Mortalidad fetal ......... 100.00

Mortalidad materna. ...... . 20.00

\section{RESUMEN}

Considerando la terminación del parto según el número de cesáreas en el total de las pacientes, tenemos:

\section{Con antocendente do una ce-}

sónea ...................

Parto espontáneo .. $\ldots \ldots \ldots .44$

Aplicación de tórceps .. . . 17

Cesáreás .. ... . . . . . . . . . . 34

Rupturas uterinas . . . . . . . 5

Con cintecedente de dos ceádrear ................ .

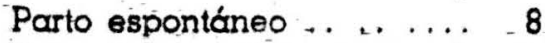

Extracción podálica ....... 1

Aplicación de fórceps ...... 3

Costrrea .. ... . . . . ..... 17

Con intecedonto do tres co. etwear ................ 7 5

5

Aplicación de fórceps - . ...

Cesárea ... ..........

Con cuntecodouts de clnco co.

Cesturea 
Teniendo en cuenta la indicación de la cesárea previa:

Desproporción

$\%$

Parto espontáneo $\ldots, \ldots, \ldots, \quad \ldots$

Aplicación de fórceps ..... 12

Cesárea ... . . . . . . . . . . 34

himitura uterina ........ 2

Eutrechez pelvica

Puto espontáneo

Extracción podálica ..... . 1

Aplicación de tórceps ... .. 2

Cesárea .............. 12

Placenta previa

Parto espontáneo ... . . . . . 9

Aplicación de fórceps ...... 2

Toxdcosis

64

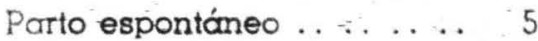

Aplicación de fórceps ...... I

Cesárea . . . . . . . . . . . 1

Ruptura uterina $\ldots \ldots \ldots \ldots$

Presentación distócica .... . 7

Parto espontáneo . . . . . . 3

Aplicación de tórceps $\ldots \ldots, 2$

Cestrea . . . . . . . . . . .

Sufrimionto fetal . . . . . . . 4

Parto espontáneo ........ 2

Aplicación de fórceps . . ., , 1

Cesárea . . . . . . . . . . 1

Primigestante eñosa ... ....

Cesárea .............. 2

Eritreblastoels fotal ... . . . .

Parto espontáneo ......... 1

Cesáred . . . . . . . .... 1

Procidoneta del cordon

Parto espontáneo ....., 2

Muerte habltual del toto ...

Cesárec ............. 1

Plastia vesleovaginal

\section{CAPITULO III}

Mortalidad fetal .......
Anoxemia por ruptura ute

rina ... . . . . . . . . . . .

Prematurez ... . . . . . .

Traumatismo obstétrico ... 2

Asfixia por retención de cabeza última $\ldots \ldots, \ldots \ldots, 1$

Eritroblastosis . . . . . . . . 1

Nacieron muertos y macerados $\ldots \ldots \ldots \ldots \ldots \ldots$

Anemia y paludismo ma: terno ............. 1

\section{CONCLUSIONES}

Como resultado del estudio hecho on 135 partos de pacientes con ante cedentes de cesárea previa, podemos sacar las siguientes conclusiones:

1. El parto espontáneo en pacientes con cesárea previa es posible.

$2^{a}$ La aplicación de fórceps en paclentes con antecedente de cesárea es conveniente como intervención proflláctica, siempre que se cumplan todas las condiciones requeridas para su correcto uso.

$3^{a}$ El parto con embarazo a término es posible por vía baja en pacienter con antecedentes de cesárec, siempre que no exiata indicación para repe tirla. 
4 Las posibilidades de un parto espontáneo en pacientes con cesárea previa, están en razón directa con el número de partos por vía baja y en razón inversa del número de cesáreas.

5 Para considerar la posibilidad de un trabajo de parto en paciente pre viamente operada de cesárea, es ne cesario conocer su historia obstétrica.

$6^{a}$ Una secundigestante con antece denté de cesárea y sin trabajo previo a la operación- debe ser considerada en su trabajo de parto como una primigestante.

$7^{a}$ Es peligroso someter a un trabajo de parto a aquellas pacientes que hicieron un puerperio tebril después del parto con cesárea.

$8^{a}$ En términos generales, cualquier paciente operada de cesárea segmentaria puede ser somenda ante un nue vo embarazo a un trabajo de parto. si existen indicaciones $y$ condiciones que lo justifiquen.

9' El trabajo de parto en pacientes con antecedente de cesćriea, debe ser julciosa e inteligentemente controlado.

10. Las -tres cuartas partes de las pacientes con antecedente de cesárea. hicieron un trabajo irregular.

11. La operación cesárea segrnentaria, cuya sola indicación es el antecedente de cesárea, sólo se ejecutó en el Instituto, en pacientes que tenian antecedente de tros cesáreas cuando menos.

12. Con el antecedente de cesćrea, una indicación de fórceps ó cesćrea, cobra un valor mucho mayor que en pacientes-que no lo tengan.

13. El número de cesáreas no justifica por sí sólo, la esterilización en ningún caso.
14. La extracción podálica es una maniobra de peligrosas consecuencias en pacientes en condiciones normales y con mayor razón en pacientes con antecedente de cesárea.

15. La versión inferna es una maniobra obstétrica que está rotunda. mente contraindicada en las pacien. tes con cesárea previa.

16. Las rupturas uterincis en pacientes con antecedente de cesárea, son debidas en gran parte, al abandonc del trabajo de parto.

\section{BDLIOGRATIA}

\section{Autores nacionales.}

1. Acomta S. Hornando - Conierencias de Obstetricia.

2. Echoverry P. Gonzalo - Consideraciones clínicas y estadisticas sobre operación cesárea. Tesis de grado de la Facultad Nacional de Medicina. 1944.

3. Forro Luls María - La operación cesárea transperitoned segmentaria. Tesis de grado de la Facul. tad Naclonal de Medicina. 1930.

4. Poralta C. Ratael - A propósito de las aplicaciones de fórceps, por encima del estrecho superior. Rev. de la Facultad Nacional de Modicina. Nং-8, Vol. 44.

5. Rodrifues Aponto Victor - Algunos aplteaciones de fórceps por encima del estrecho superior. Co municaciones a la Sociedad de Obstetricia de Colombta. Mayo de 1944. 
Autores extranjeros.

6. De Lee Greonhill - Principios y Práctica de Obstetricic:

7. Fabre - Obstetricia.

8. Dicianann William J. - Cesarean section. American Journal of Obs tetrics and Gynecology. Marzo. 1943.
9. Duckering Forence - Delivery at ter Cesarean Section. American Journal of Obstetrics and Gynecoly. Vol. 51. 1946.

10. Morogies Jaime - Clínica Obsté trica.

11. Pérez Manuel Luis - Tratado de Obstetricia.

12. Recassens - Tratado de Obsteiricia.

\section{Anotaciones sobre obstetricia rural}

\section{INFORME DE COMISION}

Señor Presidente de la Sociedad de Obstetricia y Ginecología:

La comisión designada por usted ha estudiado detenidamente el traba jo que el apreciado colega doctor Fa. raón del Portillo Ramos presentó a esta corporación en la sesión pasada, con el tin de obtener su ingress a ella.

Entre los factores que requiere un trabajo científico para permitir el acceso de su autor a esta Sociedad, la comisión manifiesta que en el presente caso ha considerado especialmente. los siguientes puntos: medio en que se efectuó la labor científica; clase de pacientes; estado generci orgánico de Las mismas en el acto operatorio; $\mathrm{cr}$. terio de la intervención; técnica qui. rúrgica; clase de anestesia; resuliados en cuanto a la madre; resultados en cuántó al niño y conclusiones gene. rales.

Como 10 anota el mismo doctor Del Portillo, on ou comunicactón, "id mit rito principal que pueden entrañar estas operaciones se refiere al medio donde han sido practicadas y sobre todo, que la mayoría de las pacientes han sido intervenidas en malas con: diciones, algunas de ellas con más de tres días de trabajo agotadas por tan Iargos sufrimientos y varias con ma nilfiestos síntomas de infección".

Se comprende perjectamente que on un medio rural y en un hospital de provincia, por bien dotado que se encuentre, la labor del médico es ardua. penosa y muchas veces estéril. Elementos de diversa indole conspiran a diario contra el buen resultado de sus

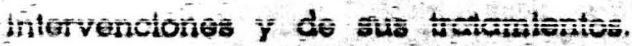
La carencia de los recursos de la torapérutica y de la cirugia modernas. considerados como de vital importan. cio on todo buen centro hospitalario, es alli un obstáculo Insalvablo parc la supervivencia de las pacientes.

Sin consulta prenalal regularmente organizada es imporible conotar bet 